\title{
Desenvolvimento e caracterização de painéis de partículas aglomeradas utilizando o resíduo do ouriço da Castanha-do-Brasil (Bertholletia excelsa) e resina poliuretana derivada do óleo da mamona
}

\author{
Development and characterization of particleboards \\ manufactured with the residue of Brazilian nut fruit \\ and castor oil polyurethane resin
}

\author{
Izaura Maria dos Santos Nogueira ${ }^{1}$, Francisco Antônio Rocco Lahr ${ }^{2}$, \\ Virginia Mansanares Giacon ${ }^{3}$
}

\author{
${ }^{13}$ Laboratório de Materiais da Amazônia e Compósitos - LaMAC - FT/UFAM - Manaus, AM. \\ e-mail: nogueiraizaura@gmail.com, e-mail: giaconbr@yahoo.com.br \\ ${ }^{2}$ Laboratório de Madeiras e Estruturas de Madeiras - LaMEM - EESC/USP - São Carlos, SP \\ e-mail: frocco@sc.usp.br
}

\section{RESUMO}

O presente trabalho teve como objetivo avaliar a viabilidade da produção de painéis de partículas aglomeradas utilizando-se o resíduo obtido após o beneficiamento do ouriço da Castanha-do-Brasil (Bertholletia excelsa) associado à resina poliuretana derivada do óleo da mamona. Um estudo das variáveis granulometria das partículas e quantidade de resina foi realizado, com base em um planejamento experimental $2^{2}+1$. A qualidade dos painéis produzidos foi avaliada com base nas prescrições do documento normativo ABNT NBR 14810:2 (2002), por meio de ensaios de absorção de umidade (2 e 24h), inchamento em espessura (2 e $24 \mathrm{~h})$ e arrancamento de parafuso (topo e superfície), adicionalmente, realizou-se um ensaio de dureza Janka e análise do teor de cinzas. Com os resultados obtidos, foi possível verificar que a incorporação de resíduo do ouriço da Castanha-do-Brasil apresenta potencial na produção de painéis de partículas aglomeradas, e que a adição de resina poliuretana à base do óleo da mamona mostrou-se eficiente como adesivo aglomerante, indicando que maiores porcentagens de resina tendem a implicar em diminuição da absorção de água e variação dimensional dos painéis, assim como aumento da resistência mecânica.

Palavras-chave: Painéis de partículas, resíduos lignocelulósicos, poliuretano natural.

\section{ABSTRACT}

The present study aimed to evaluate the potential use of Brazil nut residue in the manufacture of particleboards using castor oil polyurethane adhesive. The experimental factor investigated consisted of resin content and particle size, leading to a factorial design of $2^{2}+1$. The quality of the panels was evaluated based on determination of water absorption (WA), thickness swelling (TS) and screw withdrawal resistance, according to the Brazilian ABNT NBR 14810:2 (2002) standard, additionally, a Janka hardness and ash content tests were performed. The results demonstrated that the incorporation of Brazil nut residue has potential in the production of particleboards, and that the addition of castor oil polyurethane resin proved effective as a binder adhesive, indicating that higher percentages of the resin tend to cause low water absorption and dimensional changes in the panels, as well as higher strength values.

Keywords: Paticleboards, lignocellulosic residues, natural polyurethane.

1. INTRODUÇÃO

O processo de industrialização no Brasil teve, entre seus resultados, a destruição de riquezas naturais, o comprometimento de inúmeras espécies da fauna e da flora e fortes níveis de degradação ambiental [1]. Tais circunstâncias contribuíram para aumentar consideravelmente a preocupação em relação à preservação de nossos recursos naturais, destacando-se principalmente a exploração madeireira de nossas florestas [2]. Segundo 
BELINI [3], o crescente aumento do consumo de madeira no Brasil e no mundo desafia a sociedade atual a desenvolver produtos e tecnologias sustentáveis, que venham a substituir a madeira maciça em diversas aplicações, como na fabricação de móveis e elementos utilizados na indústria de construção civil.

Dentre os produtos derivados da madeira, os painéis de partículas aglomeradas vêm apresentando uma das maiores taxas de crescimento no mercado atual, possibilitando geração de empregos e tecnologias [4]. De acordo com dados da IBÁ [5], o Brasil produziu cerca de 7,5 milhões de $\mathrm{m}^{3}$ de painéis em 2015, percentual este ainda aquém das possibilidades de produção de nossas indústrias, considerando-se o potencial madeireiro de nosso país e as tecnologias instaladas.

A utilização de resíduos lignocelulósicos na produção de painéis de madeira tem sido objeto de pesquisa entre diversos autores, podendo-se citar o desenvolvimento de painéis aglomerados com partículas de bagaço de cana-de-açúcar [6], fibras de bananeira [7], cascas de amendoim [8], cascas de aveia [9], entre outras.

A Castanha-do-Brasil (Bertholletia excelsa), uma das espécies arbóreas de maior importância econômica na região amazônica, apresenta uma madeira de excelente qualidade, porém com proibições quanto ao seu corte. Seu fruto, popularmente chamado de ouriço, apresenta rigidez natural e caracteriza-se como resíduo de atividades extrativistas. Em estudo publicado por FAUSTINO e WADT [10], foi realizada a caracterização mecânica do ouriço da Castanha-do-Brasil por meio de ensaios de dureza Janka e resistência à compressão, obtendo valores que expõe um horizonte promissor quanto ao aproveitamento dos frutos da castanheira como carga (reforço) em painéis de madeira.

MELLO [11] verificou a resistência à abrasão de painéis aglomerados reforçados com resíduo do ouriço da castanha do Brasil utilizando a resina poliuretana derivada do óleo da mamona como adesivo, constatando que o material obteve baixo índice de desgaste, tornando viável seu uso na fabricação de pisos.

Uma vez que a produção anual média de ouriços no Brasil é abundante, girando em torno de 40 ton/ano [12], e que até então as únicas utilidades reservadas a este material estão na confecção de artesanato e carbonização para combustível em fornalhas e caldeiras [13], torna-se conveniente avaliar a aplicação desse resíduo vegetal no desenvolvimento de painéis de partículas aglomeradas.

Nesse contexto, o presente trabalho teve como objetivo o desenvolvimento de painéis aglomerados fabricados com o resíduo do ouriço da Castanha-do-Brasil associados à resina poliuretana derivada do óleo da mamona, avaliando sua viabilidade técnica através da determinação de suas propriedades físicomecânicas.

\section{MATERIAIS E MÉTODOS}

Na confecção dos painéis, empregaram-se $5 \mathrm{~kg}$ de ouriços de Castanha-do-Brasil (figura 1a), coletados nas dependências da Reserva Sauim Castanheiras, localizada na Alameda Cosme Ferreira, s/n, Distrito Industrial de Manaus II. Para estabelecer a adesão entre as partículas, foram utilizados diferentes teores de resina bi componente poliuretana derivada do óleo de mamona (figura 1b), fornecida pela Plural Indústria e Comércio de Produtos Químicos Ltda, situada na cidade de São Carlos, em São Paulo. A resina utilizada é constituída dos seguintes componentes:

- Pré-polímero (LECOPOL E 0921) derivado do petróleo, identificado como um líquido viscoso que possui aspecto marrom escuro;

- Poliol (LECOPOL F 0911) oriundo do óleo da mamona, com coloração amarelado.

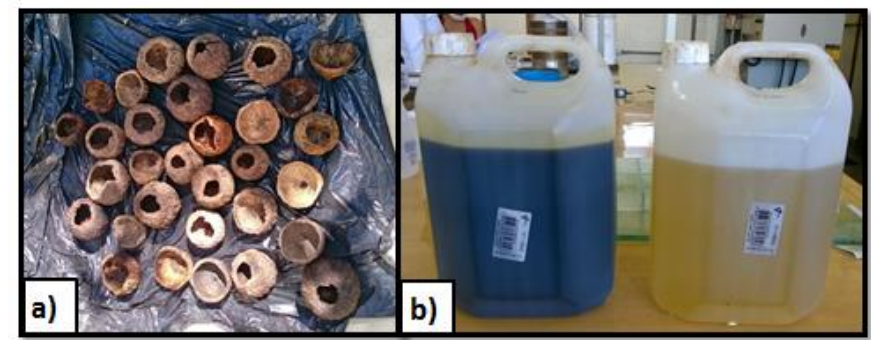

Figura 1: Principais matérias-primas utilizadas na fabricação dos painéis. 


\section{METODOLOGIA}

Inicialmente, foram realizados estudos preliminares para determinação dos critérios a serem adotados no processo de desenvolvimento dos painéis, como as porcentagens de resina a serem utilizadas e o teor de partículas lignocelulósicas. Teores de resina poliuretana derivada da mamona inferiores a $15 \%$ foram previamente experimentados, no entanto, os painéis produzidos não apresentaram propriedades mecânicas significativas, dessa forma, estipulou-se o uso de teores de resina de $20 \%, 25 \%$ e $30 \%$. Após esta fase, deu-se início ao processo de fragmentação da matéria-prima e produção dos painéis, no Laboratório de Materiais da Amazônia e Compósitos - LaMAC, da Universidade Federal do Amazonas. Nos itens abaixo, são descritas as etapas para o desenvolvimento do trabalho.

\subsubsection{Obtenção do resíduo do ouriço de Castanha do Brasil}

Esta etapa da pesquisa compreendeu a coleta, limpeza e beneficiamento dos ouriços de castanha, conforme mostra a figura 2 :

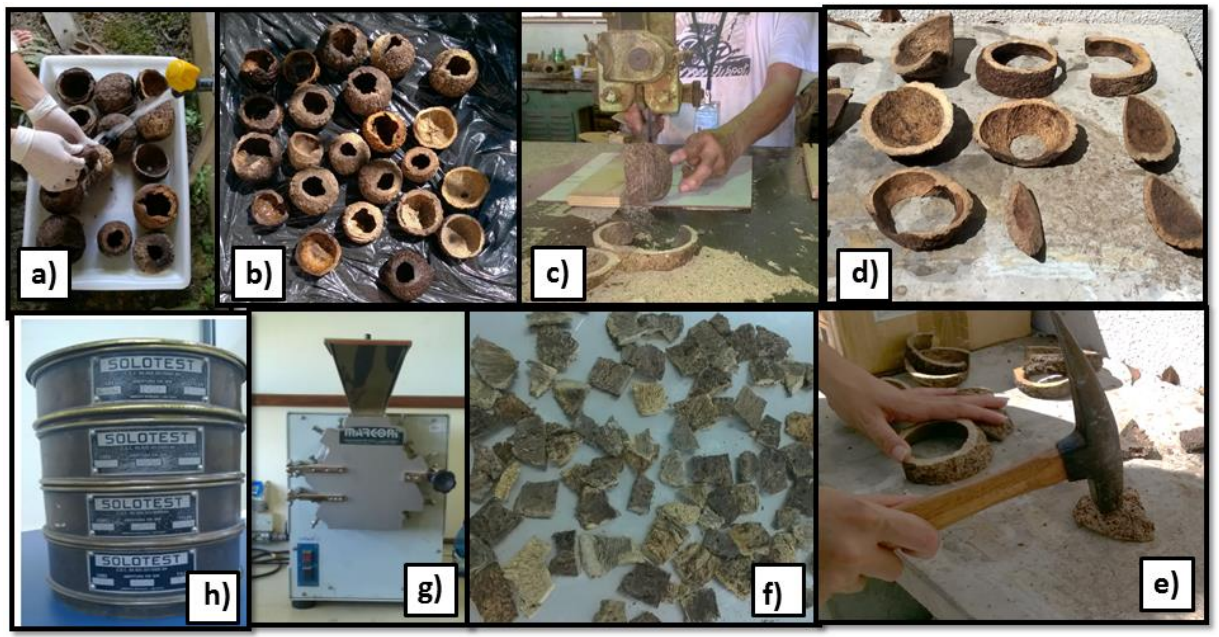

Figura 2: Processo de beneficiamento do ouriço.

Após a limpeza do material com água corrente e secagem (figuras 2a e 2b), o mesmo foi fragmentado em pedaços menores, de modo a se adequar ao equipamento utilizado na trituração. Tal ação consistiu na serragem dos ouriços, com o auxílio de uma serra de fita (figura 2c), para a obtenção de tiras (figura 2d), e posterior uso de martelo (figura 2e) para que a dimensão desejada fosse atingida (figura $2 \mathrm{f}$ ).O processo de trituração foi realizado utilizando-se um moinho macro de rotor vertical com facas móveis e fixas (figura $2 \mathrm{~g}$ ), modelo MA 680, da marca Marconi. Após a moagem, o material particulado passou por procedimento de classificação granulométrica, utilizando-se um jogo de peneiras (figura $2 \mathrm{~h}$ ).

\subsubsection{Planejamento experimental}

Com o intuito de verificar a influência dos fatores teor de resina, granulometria da partícula e da interação entre ambos nas propriedades físicas e mecânicas dos painéis produzidos, foi delineado um planejamento fatorial $2^{2}+1$, com o auxílio do software STATISTICA ${ }^{\circledR}$, versão 12.0. Tal planejamento conta com um ponto central (amostra P3), o qual é executado em triplicata, com o propósito de fornecer uma estimativa do erro experimental. Todos os resultados obtidos foram submetidos à análise de variância (ANOVA), a um nível de 95\% de confiabilidade.

A tabela 1 apresenta a composição e o número de painéis desenvolvidos nesta pesquisa, baseados em um planejamento fatorial $2^{2}+1[14]$ :

Tabela 1: Composição dos painéis baseado no planejamento fatorial $\left(2^{2}+1\right)$. 


\begin{tabular}{|c|c|c|c|}
\hline $\begin{array}{l}\text { TEOR DE RESINA } \\
\text { (\%) }\end{array}$ & $\begin{array}{l}\text { CLASSIFICAÇÃO } \\
\text { GRANULOMÉTRICA } \\
\text { (MESH) }\end{array}$ & IDENTIFICAÇÃO & $\begin{array}{l}\text { QUANTIDADE DE } \\
\text { PAINÉIS } \\
\text { PRODUZIDOS }\end{array}$ \\
\hline 30 & 14 & P1 & 1 \\
\hline 30 & 48 & $\mathrm{P} 2$ & 1 \\
\hline 25 & MIX * & P3 & 3 \\
\hline 20 & 14 & $\mathrm{P} 4$ & 1 \\
\hline 20 & 48 & P5 & 1 \\
\hline
\end{tabular}

*MI $\overline{\mathbf{X}}=50 \%$ de partículas mesh 100 e 200 (pó), $25 \%$ de partículas mesh 14 e $25 \%$ de partículas mesh 48

\subsubsection{Produção dos painéis aglomerados}

Para a fabricação dos painéis, foi utilizado o procedimento adotado por QUIRINO [15], conforme descrito a seguir. Adicionou-se a resina bi componente derivada do óleo da mamona ao resíduo obtido do beneficiamento dos ouriços. O adesivo foi acrescido em diferentes teores $(20 \%, 25 \%$ e $30 \%)$ em relação à massa seca das partículas ( $\pm 630,00 \mathrm{~g}$ ), em proporção de 1:1 (figura 3b). A mistura (figura 3c) posteriormente foi alocada em um molde (figura 3d) de dimensões $28 \times 28 \times 1 \mathrm{~cm}$ para a pré-compactação em uma prensa mecânica (figura $3 \mathrm{e}$ ), por 5 minutos, a uma pressão de 0,5 MPa. Logo após, foi realizada a prensagem a quente do material, em um prensa hidráulica térmica (figura 3f) da marca Hidral-Mac, modelo PHH 11007, em temperatura de aproximadamente $110^{\circ} \mathrm{C}$, por 10 minutos, a uma pressão de compactação de $15 \mathrm{MPa}$. O produto final (figura $3 \mathrm{~g}$ ) passou por um processo de cura de 72 horas.

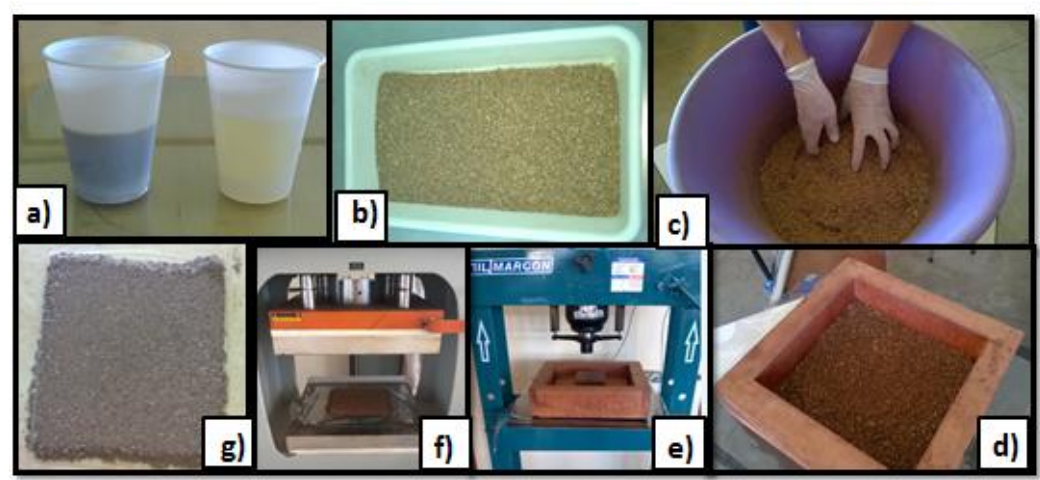

Figura 3: Processo de manufatura dos painéis.

\subsubsection{Métodos para a avaliação do desempenho}

\subsubsection{Teor de cinzas}

A determinação dos componentes minerais presentes na estrutura das fibras do ouriço da Castanha-do-Brasil foi realizada por meio do ensaio de Teor de Cinzas, seguindo as especificações da norma TAPPI T15 m-58 [16].

\subsubsection{Caracterização físico-mecânica dos painéis}

As propriedades físico-mecânicas dos painéis produzidos foram avaliadas por meio de ensaios de absorção e inchamento ( 2 e 24 horas), arrancamento de parafuso (topo e superfície) e dureza Janka, de acordo com os documentos normativos ABNT NBR 14810:2 (2002) [17] e ANSI A208:1 [18].

\section{RESULTADOS}




\section{TEOR DE CINZAS}

A tabela 2 apresenta os resultados da análise de teor de cinzas realizada no resíduo obtido do beneficiamento do ouriço da castanha.

Tabela 2: Resultados da análise de teor de cinzas.

\begin{tabular}{l|l}
\hline TEOR DO COMPONENTE (\%) & MÉDIA E DESVIO PADRÃO \\
\hline Cinzas & $1,00 \pm 0,20$ \\
\hline
\end{tabular}

Segundo Morais et al. [18], os compostos minerais (cinzas) encontrados nas madeiras em geral correspondem a valores que variam de $0,1 \%$ a $1,0 \%$. O valor médio do teor de cinzas obtido neste trabalho foi de $1 \%$.

\section{CARACTERIZAÇÃO FÍSICO-MECÂNICA DOS PAINÉIS}

A tabela 3 apresenta os valores médios obtidos através das análises físicas de absorção de água (AB) e inchamento em espessura (IE), em 2 e 24 horas, nos painéis aglomerados.

Tabela 3: Propriedades físicas dos painéis.

\begin{tabular}{l|l|l|l|l}
\hline COMPOSIÇÃO DO & AB 2H & AB 24H & IE 2H & IE 42H \\
PAINEL & $(\%)$ & $(\%)$ & $(\%)$ & (\%) \\
\hline P1 & $0,93 \pm 0,27$ & $6,30 \pm 0,29$ & $0,79 \pm 0,15$ & $3,18 \pm 0,16$ \\
\hline P2 & $3,05 \pm 0,41$ & $11,91 \pm 0,34$ & $1,07 \pm 0,09$ & $3,48 \pm 0,10$ \\
\hline P3 & $4,98 \pm 0,55$ & $17,63 \pm 0,40$ & $1,50 \pm 0,16$ & $4,10 \pm 0,14$ \\
\hline P4 & $12,92 \pm 0,60$ & $22,80 \pm 0,60$ & $2,02 \pm 0,12$ & $5,52 \pm 0,11$ \\
\hline P5 & $5,95 \pm 0,28$ & $22,09 \pm 0,19$ & $2,75 \pm 0,14$ & $6,57 \pm 0,14$ \\
\hline NBR 14810:1 (2002) & NA & NA & 8 & NA \\
\hline
\end{tabular}

*NA= Não aplicável

Como pode ser observado, com a redução da quantidade de resina na composição dos painéis, há uma tendência ao aumento da absorção de água e teor de umidade. Os painéis P1 e P2, confeccionados com 30\% de resina poliuretana derivada do óleo da mamona, obtiveram os menores valores destas propriedades.

A norma brasileira ABNT NBR 14810:2 (2002) estabelece somente um valor máximo aceitável de inchamento em espessura no período de $2 \mathrm{~h}$, o qual não deve ser superior a $8 \%$. Desta forma, todas as placas produzidas encontram-se dentro do requerido pela norma. A norma não estabelece valores máximos de absorção de água ( 2 e 24 horas) para painéis aglomerados.

A figura 4 exibe as curvas de nível, obtidas com o auxílio do software STATISTICA ${ }^{\circledR}$, utilizadas para verificar a influência dos fatores definidos durante o delineamento experimental, sobre as variáveis respostas absorção de umidade e inchamento em espessura: 


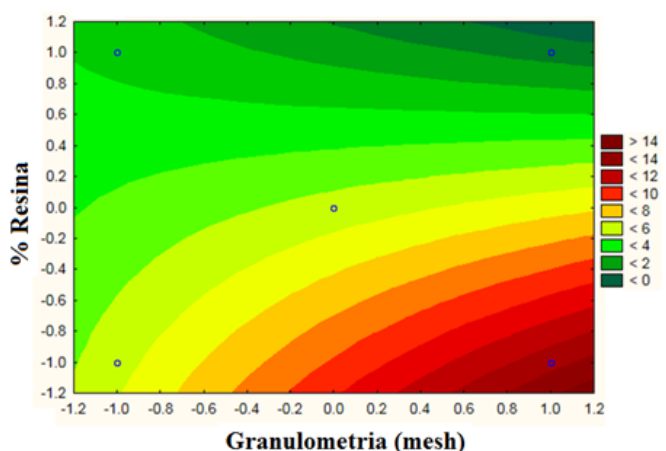

(a)

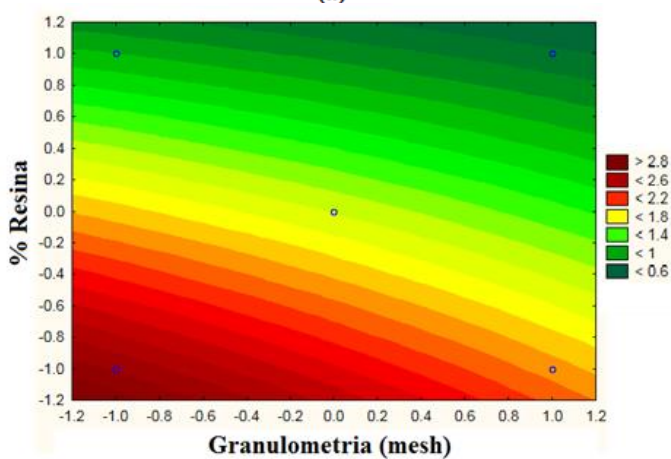

(c)

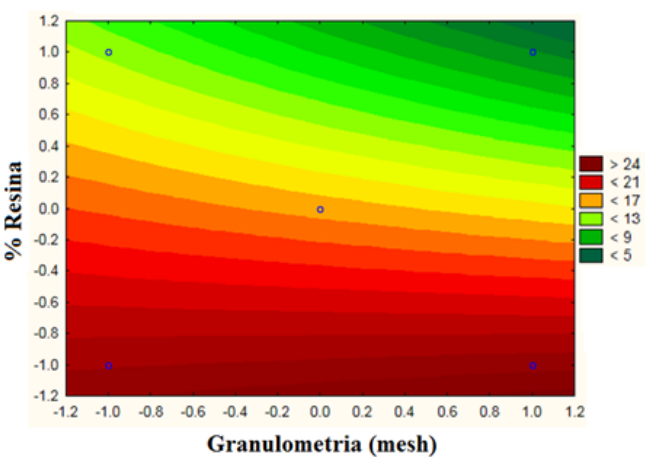

(b)

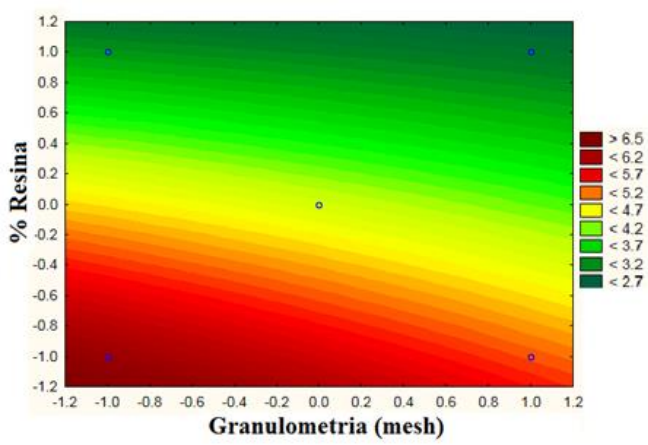

(d)

Figura 4: Curvas de nível para as variáveis dependentes (a) absorção de umidade 2h; (b) absorção de umidade 2h; (c) inchamento em espessura $2 \mathrm{~h}$ e (d) inchamento em espessura 24 horas.

Para 2 e 24 horas de imersão (figuras 4a e 4b), observa-se, com base no gráfico, que as amostras com menor quantidade de resina e partículas de dimensão maior tendem a absorver mais água. A análise estatística indicou que, nas condições de processo estudadas, o fator resina é significativo $(p<0,05)$ em 2 e 24 horas, e que a interação entre os fatores, $\%$ de resina e granulometria, é significativa para a absorção de umidade em 2 horas.

$\mathrm{Na}$ análise do inchamento em espessura, os gráficos de superfície gerados indicam que, para $2 \mathrm{~h}$ de imersão (figura 4c), as menores variações dimensionais são observadas quando maiores valores de resina são adicionados aos painéis. Para $24 \mathrm{~h}$ de imersão (figura 4d), de forma análoga ao gráfico anterior, o inchamento em espessura será menor para maiores teores de resina. Os resultados do tratamento estatístico mostraram-se significativos $(\mathrm{p}<0,05)$ para o fator resina ( 2 e 24 horas), nas condições estudadas.

Na tabela 4 constam os valores médios da propriedade mecânica de resistência ao arrancamento de parafuso (topo e superfície) estudada na presente pesquisa.

Tabela 4: Resistência ao arrancamento de parafuso dos painéis.

\begin{tabular}{l|l|l}
\hline COMPOSIÇÃO DO PAINEL & AP $_{\mathbf{T}}$ & AP \\
& $(\mathbf{N})$ & $\mathbf{( N )}$ \\
\hline P1 & $985,57 \pm 191,24$ & $1554,35 \pm 193,46$ \\
\hline P2 & $877,69 \pm 209,57$ & $1730,87 \pm 209,37$ \\
\hline P3 & $676,09 \pm 99,40$ & $1057,48 \pm 438,42$ \\
\hline P4 & $600,66 \pm 136,19$ & $1157,18 \pm 246,12$ \\
\hline
\end{tabular}




\begin{tabular}{l|l|l}
\hline P5 & $330,97 \pm 308,73$ & $956,15 \pm 243,80$ \\
\hline NBR 14810:1 (2002) & 800 & 1020 \\
\hline
\end{tabular}

Através dos valores médios apresentados na tabela 4, pode-se constatar que as amostras produzidas com maiores porcentagens de resina apresentaram um desempenho mecânico superior às demais.

A resistência ao arrancamento de parafuso no topo das amostras apresentou valor médio superior para aquelas confeccionadas com $30 \%$ de resina PU derivada da mamona com partículas de granulometria de 14 e 48 mesh, sendo estes painéis os únicos a atenderem os requisitos mínimos exigidos pela ABNT NBR 14810:2 (2002).

Já para a condição de arranque de parafuso na superfície das amostras, apenas o painel P5, confeccionado com $20 \%$ de resina e partículas de 48 mesh, não satisfez a norma.

As curvas de nível para a resistência ao arrancamento de parafuso no topo (figura 5a) e superfície (figura 5b) dos painéis indicam que partículas de resíduo de ouriço com granulometrias maiores tendem a aumentar esta propriedade.

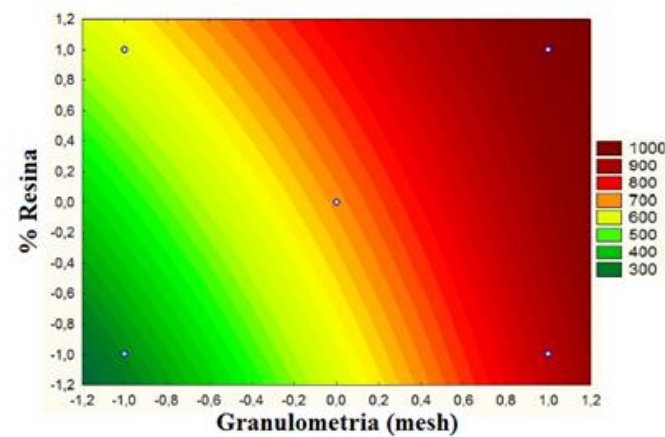

(a)

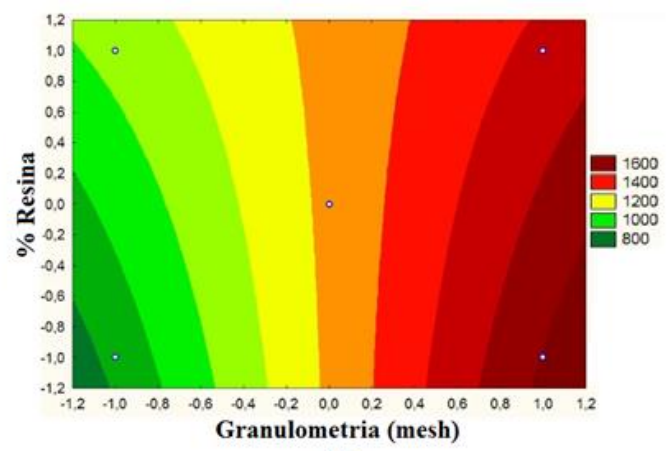

(b)

Figura 5: Curvas de nível para as variáveis dependentes (a) arrancamento de parafuso no topo da amostra e (b) superfície.

De acordo com análise de variância, para a resistência ao arrancamento de parafuso na superfície das amostras, os fatores resina e granulometria mostraram-se significativos $(\mathrm{p}<0,05)$. Já para o arrancamento de parafuso na superfície, o tratamento estatístico indicou que os fatores analisados não foram significativos nestas condições de processo.

A tabela 5 exibe os valores de dureza Janka encontrados para os painéis produzidos com o resíduo do ouriço da Castanha-do-Brasil:

Tabela 5: Valores médios de dureza Janka.

\begin{tabular}{l|l}
\hline COMPOSIÇÃO DO PAINEL & DJ \\
& (MPA) \\
\hline P1 & $69,30 \pm 20,63$ \\
\hline P2 & $74,36 \pm 25,52$ \\
\hline P3 & $53,17 \pm 27,21$ \\
\hline P4 & $51,23 \pm 15,44$ \\
\hline P5 & $40,12 \pm 19,34$ \\
\hline
\end{tabular}




\begin{tabular}{l|l}
\hline ANSI A.208 -1 (1999) & 22,25 \\
\hline
\end{tabular}

Os resultados de dureza Janka indicam que os painéis confeccionados com $30 \%$ de resina possuem resistência superficial superior àqueles confeccionados com teores $25 \%$ e $20 \%$ de adesivo. De acordo com a norma americana ANSI A.208:1 (1999), o valor mínimo exigido para painéis aglomerados é de 22,25 MPa. Deste modo, todos os painéis produzidos nesta pesquisa atenderam aos requisitos do documento normativo.

O gráfico para a variável resposta dureza Janka (figura 6a) indica que a resistência superficial dos painéis tende a aumentar conforme teores de resina superiores a $25 \%$ são empregados. $\mathrm{O}$ tratamento estatístico indicou que os fatores analisados não foram significativos, nestas condições de processo, para a dureza do material.

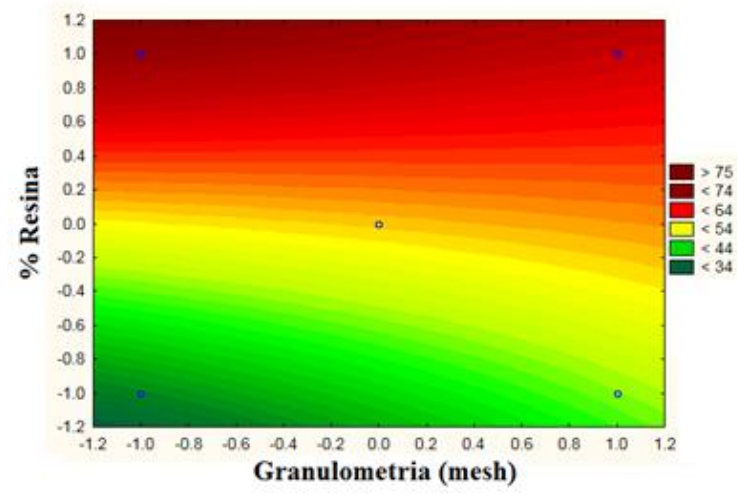

(a)

Figura 6: Curvas de nível para a variável dureza Janka.

\section{DISCUSSÃO}

A análise de teor de cinzas do resíduo estudado revelou que o teor médio destes componentes após a queima do material manteve-se de acordo com a literatura, em 1\%. LEÃO [20] analisou o teor de cinzas presente em fibras de licuri (Syagrus coronata) utilizadas como reforço em painéis de matriz polimérica, encontrando um valor de 2,86\%. GUIMARÃES [21], estudando o potencial de fibras de bananeira para aplicação em painéis aglomerados, obteve um resultado médio de 4,48\%. Altas porcentagens de cinzas resultam em alterações indesejáveis nos painéis. $\mathrm{O}$ teor de cinzas afeta o $\mathrm{pH}$ da madeira, tendendo a causar a pré-cura da resina, prejudicando assim o grau de adesão entre as partículas e provocando a redução das propriedades mecânicas dos painéis [22].

Os resultados de absorção de umidade e inchamento em espessura apresentados pelos painéis com $30 \%$ de adesivo em sua composição podem estar relacionados à característica hidrofóbica da resina poliuretana da mamona, a qual, conforme ZAU et al. [23], reduz a susceptibilidade das partículas à absorção de umidade, uma vez que preenchem as cavidades das células lignocelulósicas, tornando-as altamente resistentes à absorção de água [24]. Resultados semelhantes foram encontrados por BERTOLINI et al. [25], avaliando as propriedades físicas mencionadas acima, em painéis aglomerados confeccionados com resíduos de podas de árvores das espécies Jatobá (Hymenaea stilbocarpa) e Canelinha (Nectandra lanceolata), obtendo resultados satisfatórios, de $6,55 \%, 7,13 \%$ e 7,22\%, para absorção de água, e 4,95\%, 4,65\% e 5,58 para o inchamento em espessura, ambos em 2 horas. GAVA et al. [26] produziu painéis utilizando partículas obtidas de clones de Seringueira (Hevea brasiliensis) e $12 \%$ de adesivo poliuretano derivado da mamona, alcançando valores de absorção de água acima de 10\% para o período de 2 horas, e acima de 30\% em 24 horas, quanto ao inchamento em espessura, os autores obtiveram resultados abaixo de $4 \%$ e $17 \%$ nos períodos de 2 e 24 horas, respectivamente.

Quanto à variação dimensional dos painéis confeccionados neste trabalho, pode ser concluído que, quando partículas de geometrias menores são empregadas na confecção dos painéis, chapas com menores quantidades de espaços vazios em seu interior são produzidas, diminuindo assim sua variação dimensional [27].

Observou-se ainda que, de modo geral, com a redução da quantidade de resina nos painéis, ocorre um aumento nos valores das propriedades físicas estudadas, o que é ocasionado pela redução do efeito hidrofóbi- 
co do adesivo poliuretano, realçando as características hidrofílicas das partículas lignocelulósicas [28].

Quanto à avaliação de suas propriedades mecânicas, os resultados mostram que os painéis com maiores quantidades de resina em sua composição exibiram os melhores valores de resistência ao arrancamento de parafuso (topo e superfície). NASCIMENTO et al. [29], avaliando a qualidade de chapas produzidas com resíduos de madeira da caatinga do nordeste do Brasil e coladas com resina poliuretana à base de mamona, encontraram valores de resistência ao arrancamento de parafuso na superfície das amostras variando de $85 \mathrm{~N}$ a 140 N. BELINI et al. [30] utilizaram partículas de bambu como reforço em painéis multicamadas de bagaço de cana-de-açúcar aglutinados com $12 \%$ resina poliuretana a base de mamona, obtendo valores médios de resistência ao arrancamento de parafuso entre 637 N e 695 N, respectivamente. Em ambos os estudos, os valores obtidos situaram-se abaixo dos apresentados neste trabalho, corroborando assim com a suposição de PEDRAZZI et al. [30], a qual estabelece que, com o aumento do teor de resina, os valores de resistência ao arrancamento de parafuso sofrem um incremento.

Os valores médios de dureza Janka neste trabalho variaram de 4,01 a 7,43 $\mathrm{MPa}$, mantendo-se de acordo com o constatado por MURAKAMI et al. [31] em seu estudo sobre os efeitos do tipo e teor de resina em painéis aglomerados, os quais apresentam maior dureza superficial conforme adicionados maiores quantidades de adesivo à sua composição. De acordo com PIERRE [32], a dimensão das partículas também influencia nesta propriedade, pois quando partículas de menor granulometria são comprimidas para a produção do painel, ocorre um maior contato relativo entre elas, originando a maior adesão das partículas, e consequentemente maior resistência mecânica.

\section{CONCLUSÕES}

Baseando-se nos resultados obtidos, são apresentadas as seguintes conclusões:

- É possível a utilização de resíduos do ouriço da Castanha-do-Brasil na fabricação de painéis de partículas aglomeradas utilizando teores de $20 \%, 25 \%$ e $30 \%$ de resina poliuretana à base de mamona;

- Através da análise do teor de cinzas presente nas partículas, o ouriço da Castanha-do-Brasil apontou ser um resíduo florestal com potencial para fabricação de painéis, uma vez que exibe percentual de componentes minerais dentro dos valores encontrados na bibliografia;

- Observou-se que, nas condições de processo estudadas, teores de resina de $30 \%$ proporcionaram melhores resultados de propriedades físicas e mecânicas nos painéis fabricados;

- Considerando-se o desempenho satisfatório obtido através da caracterização físico-mecânica, é possível concluir que o material apresenta boas propriedades de variação dimensional e resistência mecânica, comprovando assim seu potencial de utilização nos setores de construção civil e moveleiro.

\section{AGRADECIMENTOS}

Ao Laboratório de Madeiras e Estruturas de Madeiras (LaMEM) da Escola de Engenharia de São Carlos USP.

\section{BIBLIOGRAFIA}

[1] BAUMGARTEN, M. "Sociedade e Sustentabilidade: qual o lugar do conhecimento?", Sociologias, v. 16, n. 37, pp. 14-22, Dez. 2014.

[2] POLETO, S.F.S., BERTOLINI, M.F., NASCIMENTO, M.F., et al. "Produção de Chapas de Partículas homogêneas (CPH) utilizando resíduos de espécies de reflorestamento", Reciclagem de resíduos para a construção civil, Belo Horizonte, FUMEC-FEA, v. 01, pp. 271-292.2007.

[3] BELINI, U. L., SAVASTANO JÚNIOR, H., BARRERO, N. G., et al., "Painel multicamada com reforço de partículas de bambu", Scientia Forestalis, v. 42, n. 103, pp. 421-427, Set. 2014.

[4] CAMPOS, C. I., LAHR, F. A. R. "Estudo comparativo dos resultados de ensaio de tração perpendicular para MDF produzido em laboratório com fibras de pinus e de eucalipto utilizando uréia-formaldeído", Matéria, v. 9, n. 1, pp. 32-42, 2004.

[5] Indústria Brasileira de Árvores - IBÁ. "Relatório IBÁ 2015”. Brasília. 2015.

[6] SARTORI, D. L. Painel portante estrutural com chapa de partículas de bagaço de cana-de-açúcar e resina de mamona para centro de manejo bovino. Tese de D.Sc., Universidade de São Paulo, 2012. 
[7] MERLINI, C. Análise experimental de compósitos de poliuretano derivado de óleo de mamona e fibras de bananeira, Dissertação de Mestrado, Universidade Federal de Santa Catarina, 2011.

[8] BARBIRATO, G. et al. "Crowded hybrid panel manufactured with peanut hulls reinforced with Itaúba wood particles", Ciência Florestal, v. 24, n. 3, pp. 685-697, Set. 2014.

[9] VARANDA, L. D. Produção e avaliação do desempenho de painéis de partículas de Eucalyptus grandis confeccionados com adição de casca de aveia, Tese de D.Sc., Universidade de São Paulo, 2012.

[10] FAUSTINO, C. L., WADT, L.O. "Resistência mecânica do pericarpo de frutos de Bertholletia excelsa Bonpl.(Lecythidaceae)", Revista Ciência da Madeira (Brazilian Journal of Wood Science), v. 5, n. 1, pp. 25 33, Maio. 2014.

[11] MELLO, A.K.S. Design de tecnologia social: Reaproveitamento do ouriço da castanha do Brasil no desenvolvimento de novos materiais. Dissertação de M.Sc., Pontifícia Universidade Católica do Rio de Janeiro. 2013.

[12] COMPANHIA NACIONAL DE ABASTECIMENTO, "Castanha do Brasil”, Companhia Nacional de Abastecimento, abril 2012. Brasília, CONAB, 2012

[13] CARMARGO, F. F. Etnoconhecimento e Variabilidade Morfológica de Castanha-do-Brasil (Berthollethia excelsa Bonpl.:Lecythidaceae) em Área da Amazônia Matogrossense, Dissertação de Mestrado. Universidade Federal do Mato Grosso. 2010.

[14] BARROS, B., SCARMINIO, I. S., BRUNS, R. E. Como fazer experimentos, 2 ed., Editora Unicamp, 2001.

[15] QUIRINO, M. G. Estudo de matriz polimérica produzida com resina natural e fibra da semente de açaí (Euterpe precatória), Dissertação de M.Sc., Universidade Federal do Amazonas, 2010.

[16] TECHNICAL ASSOCIATION OF THE PULP AND PAPER INDUSTRY. "Ash in Wood and Pulp: T15 om-58." Atlanta, 1991.

[17] ASSOCIAÇÃO BRASILEIRA DE NORMAS TÉCNICAS. NBR 14810-2: "Painéis de partículas de média densidade, parte 2: Requisitos e métodos de ensaio”, Rio de Janeiro, 2002.

[18] AMERICAN NATIONAL STANDARD INSTITUTE. "Particleboards: requirements and test methods". Gaithersburg, 1999.

[19] MORAIS, S. A. L., NASCIMENTO, E. A. MELO, D.C. “Análise da madeira de Pinus oocarpa parte I: estudo dos constituintes macromoleculares e extrativos voláteis", Revista Árvore, v. 29, n. 03, p. 461-470, 2005 .

[20] LEÃO, M. A. Fibras de licuri: um reforço alternativo de compósitos poliméricos. Dissertação de Metrado, Universidade Federal do Rio Grande do Norte, 2008.

[21] GUIMARÃES, B. M. R. Tratamento químico de partículas de pseudocaule da bananeira visando à produção de painéis aglomerados, Dissertação de M.Sc., Universidade Federal de Lavras, 2012.

[22] IWAKIRI, S., KEINERT JR., S., MENDES, L. M. "Painéis de madeira compensada", In: IWAKIRI, S. Painéis de madeira reconstituída. Curitiba, Fupef. 254p, 2005.

[23] ZAU, M. D. L. et al. “Avaliação das Propriedades Química, Física e Mecânica de Painéis Aglomerados Produzidos com Resíduo de Madeira da Amazônia-Cumaru (Dipteryx Odorata) e Resina Poliuretana à Base de Óleo de Mamona”, Polímeros, v. 24, n. 6, pp. 726-732, Jun. 2014.

[24] BERTOLINI, M. S. Emprego de resíduos de Pinus sp tratado com preservante CCB na produção de chapas de partículas homogêneas utilizando resina poliuretana à base de mamona, Tese de D.Sc., Universidade de São Paulo, 2011.

[25] BERTOLINI, M., NASCIMENTO, M.F., BLECHA, K.A., et al. "Eco-panels based on wastes from urban trees and castor oil Polyurethane resin", International Journal of Agriculture and Forestry, v. 3, n. 1, pp. $12-15,2013$.

[26] GAVA, M., MUZEL, S.D., LIMA, L.R., et al., "Production of Particleboards from Hevea brasiliensis Clones and Castor Oil-based Polyurethane Resin", BioResources, v. 10, n. 4, pp. 6896-6905, Ago. 2015.

[27] VITAL, B. R., HASELEIN, C. R., DELLA LUCIA, R. M. "Efeito da geometria das partículas nas propriedades dos painéis de madeira aglomerada de Eucalyptus grandis (Hill ex-Maiden)", Revista Árvore, Viçosa, v. 16, n. 1, pp. 88-96, 1992. 
[28] ABREU, A. L. Modificação química de resíduo lignocelulósico para a preparação de compósito. Dissertação de Mestrado, Universidade Federal de Lavras, 2011.

[29] NASCIMENTO, M. F., BERTOLINI, M.S., PANZERA, T.H., et al. "Painéis OSB fabricados com madeiras da caatinga do nordeste do Brasil”, Ambiente Construído, v. 15, n. 1, pp. 41-48, 2015.

[30] PEDRAZZI, C., HASELEIN, C.R., SANTINI, E.J., et al., "Qualidade de chapas de partículas de madeira aglomerada fabricadas com resíduos de uma indústria de celulose”, Ciência Florestal, Santa Maria, v. 16, n. 2, pp. 201-212, abr./jun. 2006.

[31] MURAKAMI, K., MINORU, U., HIDEAKI, M., et al., "Manufacture and properties of three-layered particleboards with oriented face strands of veneers: 1", Japan Wood Science, Tokyo, v.45, n.5, pp.395-402, 1999

[32] PIERRE, F. C. Caracterização físico-mecânica de painéis aglomerados de Eucalyptus grandis com adição de resíduos industriais madeireiros, Tese de D.Sc., Universidade Federal de Lavras, 2010. 